\title{
Design and Development of a High Performance Pinhole Array Dual Head SPECT Using Monte Carlo Simulation
}

\author{
Tasneem Rahman, Murat Tahtali, and Mark R. Pickering
}

\begin{abstract}
The Purpose of this study is to investigate the performance of a dual head SPECT system having an array of pinholes as a collimator. Several analytical methods are used to evaluate the performance of the given pinhole array detector module design. Monte Carlo simulation studies using Geant4 Application for Tomographic Emission (GATE) validate the performance of this novel dual head SPECT where a cylindrical water phantom is imaged. We are proposing a pinhole array detector module with $48 \mathrm{~mm}$ by $48 \mathrm{~mm}$ of active area behind an array of 100 pinholes laid out in a 10 by 10 lattice. This configuration allows high detection efficiency and excellent sensitivity in SPECT imaging. The system is based on a pixelated array of $\mathrm{NaI}$ crystals $(100 \times 100 \times 10 \mathrm{~mm}$ elements $)$ coupled with an array of position sensitive photomultiplier tubes (PSPMTs). The basic characteristics of this system were evaluated with pinhole apertures of $0.5 \mathrm{~mm}, 1 \mathrm{~mm}, 1.5 \mathrm{~mm}, 2 \mathrm{~mm}$, $2.5 \mathrm{~mm}$ and $3 \mathrm{~mm}$ respectively. A conventional filtered back projection algorithm was used to reconstruct the images. Performance evaluation of this system indicates that high quality images can be obtained for in vivo imaging.
\end{abstract}

Index Terms-Dual head SPECT, SPECT instrumentation, pinhole SPECT and GATE.

\section{INTRODUCTION}

In the past several years, SPECT has developed significantly and is now considered to be an imperative modality in the nuclear emission field. SPECT is a physiological imaging tool that is commonly used for visualizing or measuring the function of specific organs. There are definite advantages for improved SPECT devices [1]. Higher sensitivity and resolution are necessary for small animal imaging than for human imaging to achieve a level of anatomic detail that is comparable to human studies. Dedicated small animal imaging systems are being developed in several modalities. In spite of the requirement for higher spatial resolution, SPECT is most often based on the Anger camera principle: a collimator is placed in front of a $\mathrm{NaI}(\mathrm{Tl})$ scintillation crystal associated with a set of photomultipliers. Conventional collimators used for SPECT imaging cannot provide better detection efficiency and result in poor image quality. SPECT is a rapidly changing field, and the past several years have produced new developments in both hardware technology and image processing algorithms [2]. At the component level, there have been improvements in scintillators and photon transducers as well as in the greater availability of semiconductor technology. The current

Manuscript received September 10, 2014; revised November 25, 2014.

The authors are with the School of Engineering and Information Technology, UNSW Australia, Canberra, Australia (e-mail: tasneem.rahman@student.adfa.edu.au, m.tahtali@adfa.edu.au, m.pickering@adfa.edu.au). state-of-the-art of SPECT has been developing from many fronts and there is still scope to see improvements in instrumentation and innovative image reconstruction algorithms. Despite the readily available isotopes, unlike PET, SPECT suffers from low detection efficiency and low resolution; therefore, many investigations have focused on increasing its sensitivity without degrading spatial resolution [3]-[5]. A pinhole approach for SPECT is envisaged for superior spatial resolution compared to conventional parallel-hole collimators [6]. A multi-pinhole collimator could provide high resolution SPECT imaging with comparable sensitivity to parallel-hole collimators. Different pinholes used in multiplexed projections provide efficient coverage of the detector area while it increases the complexity for image reconstruction [7], [8]. Several studies to employ multi-pinhole collimators have been conducted recently and have demonstrated superior performance for in vivo imaging of small animals. For preclinical systems, a multi pinhole arrangement enables positioning of the events within a cone in the field of view (FOV) and facilitates image reconstruction.

Focusing a small field of view (FOV), pinholes in the collimator generate multiplexed projections through different pinholes. In a study on a similar aspect, a stationary system dedicated to the imaging of small animals with non-multiplexed projections was proposed [9]. This system, showing sub millimeter details of tracer uptake, allows assessment of tracer dynamics in the organs of the living mouse. Several systems confirm that a multi-pinhole collimator could provide enhanced resolution and sensitivity compared to single-pinhole and parallel-hole collimators [10], [11]. However, as a result of the small FOV of about $50 \mathrm{~mm}$, the application of the system is restricted to small animal imaging. In clinical applications for myocardial perfusion imaging, a multi-pinhole collimator comprised of seven pinholes was introduced [12]. The process used planar emission tomography obtained with a limited angle using a standard gamma camera. Angular sampling, however, was insufficient to reconstruct the heart without image distortion and loss of spatial resolution. The resolution was poor due to the large pinhole diameter. For that reason, a 9-pinhole collimator was investigated to improve angular sampling of the heart by increasing the number of pinholes and acquisition angle of the detector. This 9-pinhole collimator indicates the possibility of improving detection efficiency and spatial resolution compared to parallel-hole collimators [13], [14]. Most multi-pinhole systems employ a conventional gamma camera with $3 \sim 5 \mathrm{~mm}$ intrinsic spatial resolution.

These systems use large magnifications to provide superior spatial resolution with relatively poor intrinsic spatial 
resolution. Recently, solid state detectors have been developed with spatial resolutions below $2 \mathrm{~mm}$ [15], [16]. If detectors having higher intrinsic spatial resolutions are used for pinhole systems, they could offer higher resolutions without magnification. To image radiolabeled molecules, the multi-pinhole collimator has been widely verified for its superior spatial resolution and sufficient efficiency [15]. However, most developed systems are for small objects and as a result not suitable for patient studies [17]. Hence, there is an obvious need to develop a multi-pinhole system for patient studies to provide both a high resolution and large FOV. We are proposing a new multi-pinhole design that can provide good resolution and system sensitivity compared to parallel-hole collimators. This detector module could be utilized in various investigative and clinical studies.
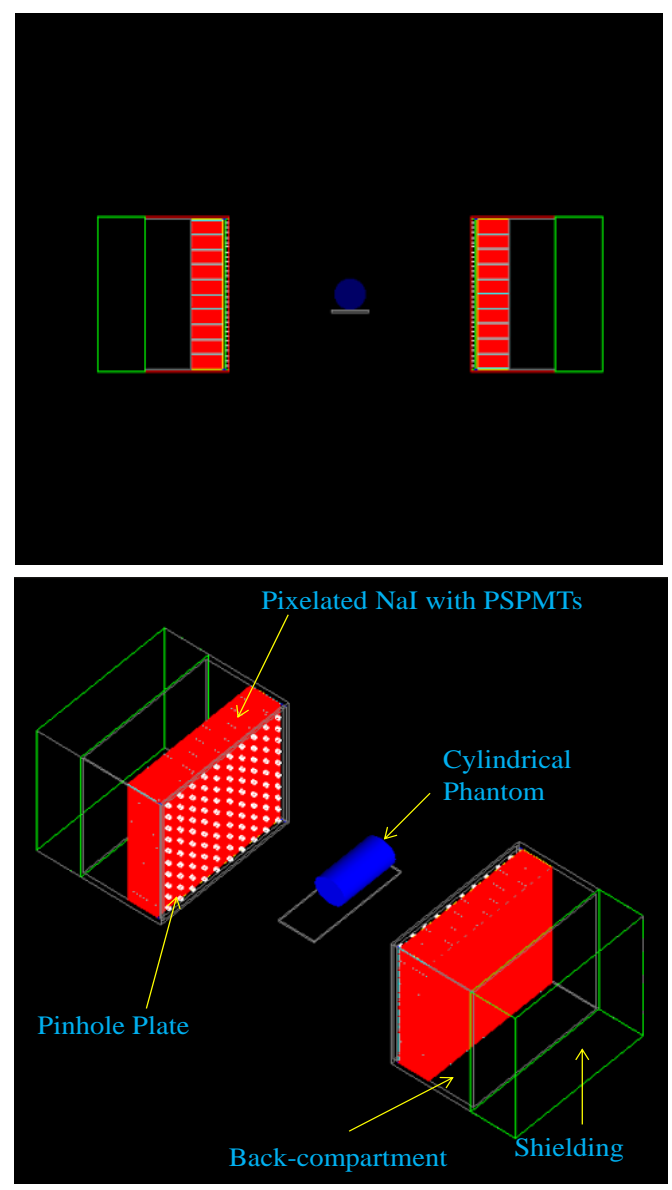

Fig. 1. Dual head pinhole array detector SPECT model.

This article describes and validates a newly developed dual head SPECT Monte Carlo simulation model. We have evaluated this newly designed system based on its sensitivity, uniformity, and scatter fraction efficiency along with its polarization characteristics. This study aims to develop an emission tomographic modality in such a way that it can produce better spatial and temporal resolutions, as well as demonstrating a non-superimposed technology while hoping to reduce scanning time. This model enables feasibility studies of new imaging applications which will expose a developed molecular imaging technology.

\section{Proposed Method}

To evaluate the performance of this newly designed dual head SPECT system, we have used Monte Carlo simulations and have observed the effect of different object sizes.

\section{A. System Description}

\section{1) Pinhole array detector module design}

The proposed SPECT subsystem contains two detector heads. Fig. 1 shows the position of both the detectors which are situated at $90^{\circ}$ and $270^{\circ}$. Each detector module is designed to have a $48 \mathrm{~mm} \times 48 \mathrm{~mm}$ active area. The detector module consists of $48 \times 48 \times 10 \mathrm{~mm}^{3}$ crystals coupled to a pinhole-array collimator. Each detector consists of a $4.8 \mathrm{~mm}$ $\times 4.8 \mathrm{~mm} \times 10 \mathrm{~mm}$ pixelated scintillator array of $0.04 \mathrm{~mm} \times$ $0.04 \mathrm{~mm} \times 10 \mathrm{~mm} \mathrm{NaI}$ crystals. The detector pixels are separated from one another by a $0.048 \mathrm{~mm}$ reflective compound. The scintillator is attached to a light guide that is attached to a 10 by 10 array of $48 \mathrm{~mm} \times 48 \mathrm{~mm}$ PSPMTs (Position Sensitive Photo Multiplier Tubes). The detectors have their back compartments with extra shielding of $48 \mathrm{~mm}$ $\times 48 \mathrm{~mm} \times 10 \mathrm{~mm}$. The maximum active imaging region is 48 $\mathrm{mm} \times 48 \mathrm{~mm}$ and the detectable energy range is from $20 \mathrm{keV}$ to $160 \mathrm{keV}$. This 100 pinhole configuration is present in each detector head. A tungsten plate of $48 \mathrm{~mm} \times 48 \mathrm{~mm} \times 1 \mathrm{~mm}$ is used as the pinhole plate as shown in Fig. 2.

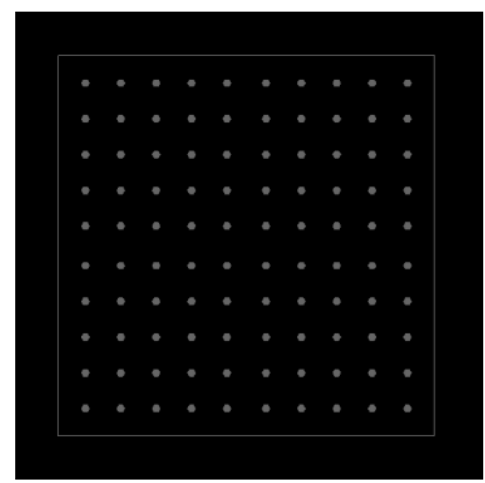

Fig. 2. Tungsten plate of pinhole array.

Pinhole characteristics were modelled for acceptance angle, pinhole diameter, depth and orientation. Pinhole diameters are in the range $0.5 \mathrm{~mm}$ to $3 \mathrm{~mm}$. Behind each pinhole, there is an array of 100 by 100 pixels with each array corresponding to a PMT, as shown in Fig. 3. All the pixels are used for generating projection images. Photon interactions within the same array are treated as being processed by the same PMT.

\section{2) Miniature phantom study}

Fig. 1 shows a uniform cylindrical water phantom. Since there are no specific standards to evaluate SPECT modality like in other imaging systems, a general water phantom was chosen to perform the initial evaluation for this dual head SPECT model. The $5 \mathrm{~mm}$ diameter by $20 \mathrm{~mm}$ height cylindrical phantom is filled with ${ }^{99 \mathrm{~m}} \mathrm{Tc}$ of $140 \mathrm{keV}$. The size of the phantom was chosen so that it is less than the total active region of a detector module.

\section{B. Monte Carlo Simulation}

GATE models are used to develop critical imaging systems as it is flexible to allow various technical experiments to execute which are not feasible in a real physical system for various reasons. GATE [18] is a Monte Carlo simulation package based on GEANT4 [19] that has 
been specifically tailored to the modelling of tomographic imaging systems. The OpenGATE Collaboration has enabled researchers to develop and share models for a number of preclinical systems. This collaborative network of developers has created the resources necessary for others to develop their own models. The Monte Carlo simulations were performed to assess the image quality of this dual-head SPECT system using GATE. GATE simulations are widely used in nuclear medicine, especially in the development of new imaging instruments, image acquisition strategies, processing and reconstruction methods. A large variety of prototype systems have been simulated using GATE. In this simulation, 140 $\mathrm{keV}$ mono-energetic gamma rays were assumed to be emitted. The physical process involving photon interactions (photoelectric effect, Compton scattering and Rayleigh scattering) were modeled using the low-energy electromagnetic package of GEANT4, while gamma conversion was disabled. The spatial resolution and sensitivity were measured using a $10 \mathrm{MBq}{ }^{99 \mathrm{~m}} \mathrm{Tc}$ point source. The dual head SPECT was simulated to reduce the acquisition time and 60 projections were obtained from $0^{\circ}$ to $360^{\circ}$ with a $6^{\circ}$ step. Fig. 1 shows the simulation configuration of the SPECT system with a pinhole array collimator.

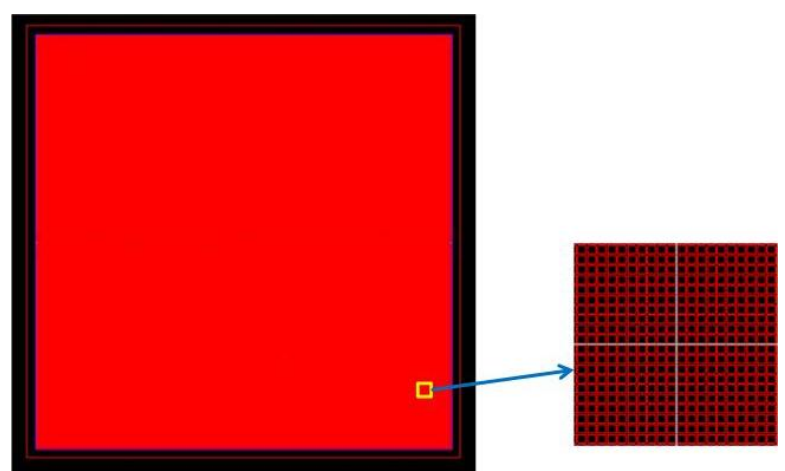

Fig. 3. Pixelated scintillation NaI array incorporated with position sensitive photo multiplier tubes (PSPMTs) has been shown in magnification section.

\section{Basic System Measurements}

\section{1) Sensitivity}

Sensitivity of the system can be defined as the division of the number of detected events per acquisition time by the number of emitted events [20]. System sensitivity was measured using a $5 \mathrm{~mm}$ diameter by $20 \mathrm{~mm}$ long cylindrical phantom containing ${ }^{99 \mathrm{~m}} \mathrm{Tc}$ source. The source was placed in the center point and sensitivity was measured at several distances and with various pinhole apertures. All the measurements were executed using a $20 \%$ photo peak energy window.

\section{2) Energy spectra and energy resolution}

The energy spectra of ${ }^{99 \mathrm{~m}}$ Tc were simulated. A uniform shape cylindrical water phantom having $5 \mathrm{~mm}$ diameter and $20 \mathrm{~mm}$ length filled with $20 \mu \mathrm{Ci}$ was used to measure the energy resolution on a pixel-by-pixel basis which is defined using the amplitude of the crystals energy spectrum. The average of the individual energy spectra can be expressed as the overall energy resolution. A 37.5 minutes acquisition session was simulated with both detectors acquiring data in a stationary position at $10 \mathrm{~cm}$ radius of rotation (RoR). The energy resolution for ${ }^{99 \mathrm{~m}} \mathrm{Tc}$ was measured by calculating the full width half maximum of the corresponding energy peaks divided by the value of the center of the photo peak.

\section{3) Scatter fraction}

The scatter fraction [21] was measured by acquiring images of the same phantom. The source was positioned along the central axis of the pinhole collimator and placed in the center of the phantom. The number of counts acquired in a fixed time interval was recorded. The measurement was repeated with the source removed from the water and placed in air at the same distance from the pinhole. The scatter fraction was measured using a $10 \%$ energy window for several pinhole apertures and with several source to pinhole distances. The total number of counts were corrected for radioactive decay and used to compute the scatter fraction for each cylinder as follows:

$$
S F=\frac{\sum W-k \sum A}{\sum W}
$$

Here $k=e^{-\mu R}, R$ is the radius of the cylinder, and $\mu$ is the attenuation coefficient $\left(0.152 \mathrm{~cm}^{-1}\right)$ for $140-\mathrm{keV}$ gamma rays in water.

4) System uniformity

The system uniformity [22] was measured using a cylindrical water source of a $20 \mu \mathrm{Ci}{ }^{99 \mathrm{~m}} \mathrm{Tc}$ source located at the central axis for 37.5 hours. Acquisition was simulated with both detectors acquiring data in a stationary position. The system detectors were positioned at $10 \mathrm{~cm}$ ROR. The data were processed to calculate the integral uniformity by following the procedures outlined in the NU-1 2007 standards [14]. The system uniformity was calculated in the following way:

$$
\left[S U(\%)=\frac{\operatorname{Max}_{c p s}-\operatorname{Min}_{c p s}}{\operatorname{Max}_{c p s}+\operatorname{Min}_{c p s}} \times 100\right]
$$

Here $\operatorname{Max}_{c p s}$ and $\operatorname{Min}_{\text {cps }}$ are the maximum and minimum counts per second, respectively.

\section{5) Confirmation of the polarization phenomenon}

The polarization phenomenon [23] causes the counting efficiency to decrease. To determine whether the polarization phenomenon occurred, the total count was measured for each SPECT projection frame. The relationship between the total count and the frame number gave the relationship between the counts over time in the SPECT acquisition.

\section{RESULTS AND DisCUSSION}

\section{A. System Sensitivity}

The sensitivity values of the dual head SPECT system obtained using a cylindrical water shape phantom are plotted in Fig. 4 for pinhole apertures of $0.5 \mathrm{~mm}, 1 \mathrm{~mm}, 1.5 \mathrm{~mm}, 2 \mathrm{~mm}$, $2.5 \mathrm{~mm}$ and $3 \mathrm{~mm}$ in diameter. The sensitivity was measured for source to detector distances of $10 \mathrm{~cm}, 12 \mathrm{~cm}, 15 \mathrm{~cm}, 18 \mathrm{~cm}$, $20 \mathrm{~cm}, 30 \mathrm{~cm}$ and $40 \mathrm{~cm}$. The sensitivities achieved with the different pinhole apertures are compared against the source to 
detector distances. As expected, greater sensitivities were achieved for larger pinholes. The sensitivity decreased with increasing source to detector distance.

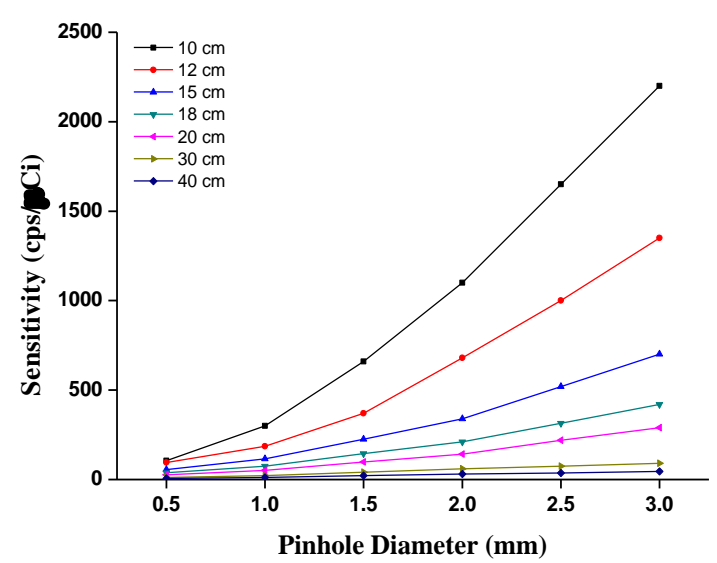

Fig. 4. Measured sensitivity based on different pinhole diameters for several source to detector distances.

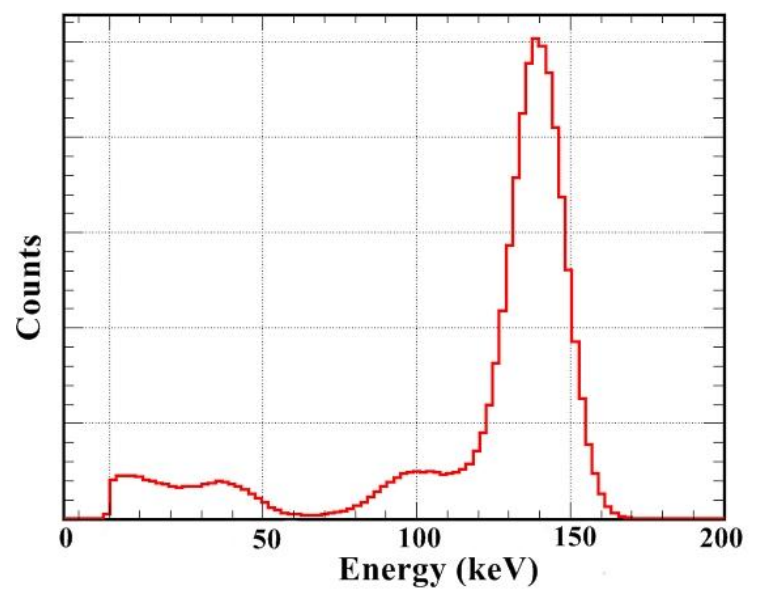

Fig. 5. Energy spectra for $140 \mathrm{keV}^{99 \mathrm{~m}} \mathrm{Tc}$ source from a single crystal.

\section{B. Energy Spectra and Energy Resolution}

Energy resolution differs from one detector pixel to another, and the mean value and standard deviation for the system were calculated to be $11.3 \pm 1.4 \%$ FWHM at $140 \mathrm{keV}$. A sample energy spectrum from one crystal is shown in Fig. 5 Reduction of the scatter count depends on the improvement of the detector energy resolution and improves the quantitative accuracy of the SPECT measurements.

\section{Scatter Fraction}

Fig. 6 shows the scatter fraction for the ${ }^{99 \mathrm{~m}} \mathrm{Tc}$ source plotted as a function of pinhole aperture in the $20 \%$ energy window for source to detector distances of $10 \mathrm{~cm}, 20 \mathrm{~cm}$ and $30 \mathrm{~cm}$. Scatter correction is important to obtain quantitative information from an image. However, from Fig. 6, it seems that scatter doesn't contribute significantly in the case of the cylindrical water phantom when the usual energy window of $20 \%$ is used.

\section{Polarization Phenomenon}

Polarization affects the performance of the system by decreasing the counting rate and charge collection efficiency. In this study, the total count of each projection for the cylindrical water phantom was almost constant. In Fig. 7, it is shown that no polarization phenomenon was observed during the 30 minutes of acquisition time, which is a substantial time for SPECT examination.

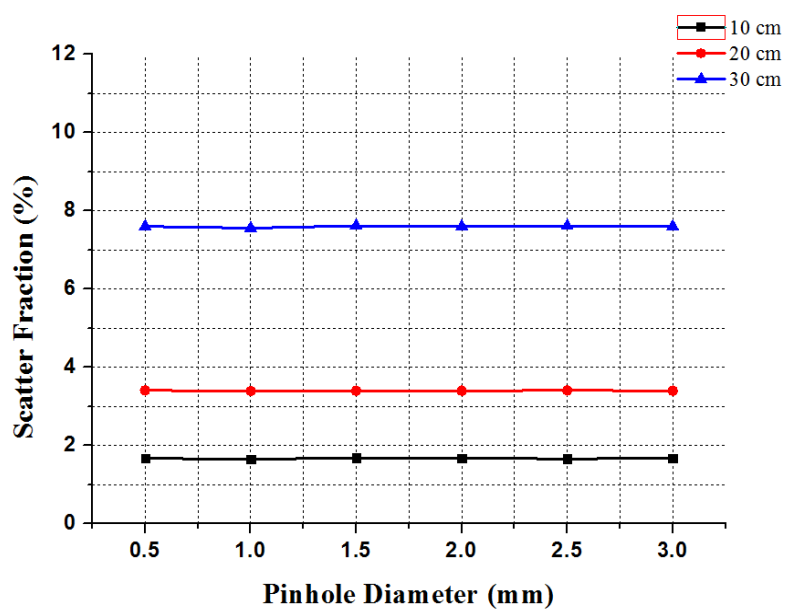

Fig. 6. Scatter Fraction as a function of several pinhole diameters.

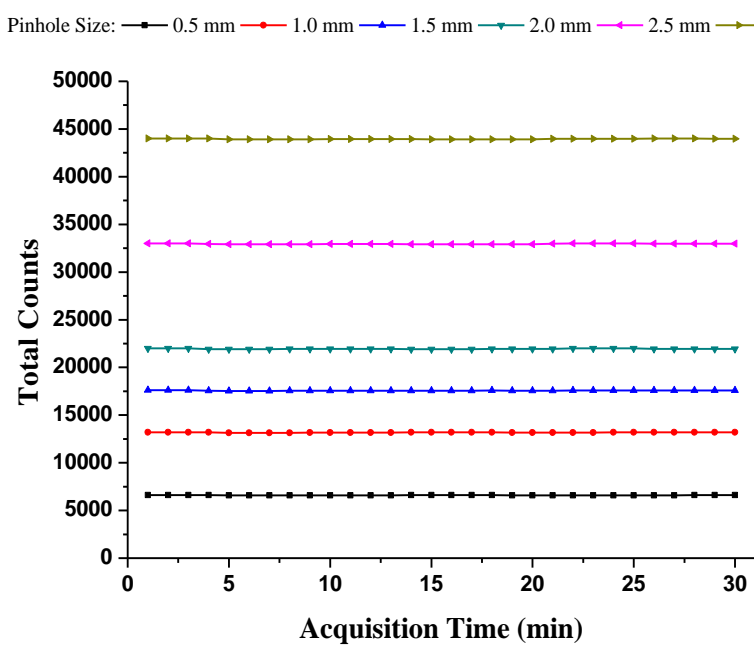

Fig. 7. Polarization phenomenon as a function of acquisition time.

\section{E. System Uniformity}

Table I shows the system uniformity for this pinhole array dual head SPECT system based on different pinhole apertures. For pinhole apertures of $0.5 \mathrm{~mm}, 1.0 \mathrm{~mm}, 1.5 \mathrm{~mm}$, $2.0 \mathrm{~mm}, 2.5 \mathrm{~mm}$ and $3.0 \mathrm{~mm}$, the system uniformity was found to be $6.12 \%, 6.32 \%, 6.70 \%, 7.69 \%, 8.04 \%$ and $8.51 \%$ respectively.

TABLE I: SYSTEM UNIFORMITY FOR DIFFERENT PINHOLE APERTURES

\begin{tabular}{cc} 
Pinhole Apertures & System Uniformity, $S U(\%)$ \\
\hline $0.5 \mathrm{~mm}$ & 6.12 \\
$1.0 \mathrm{~mm}$ & 6.32 \\
$1.5 \mathrm{~mm}$ & 6.70 \\
$2.0 \mathrm{~mm}$ & 7.69 \\
$2.5 \mathrm{~mm}$ & 8.04 \\
$3.0 \mathrm{~mm}$ & 8.51 \\
\hline \hline
\end{tabular}

\section{F. Phantom Image}

Fig. 8 shows the projection image and Fig. 9 shows the reconstruction slices for different projections acquired during one run of the experiment where the uniform cylindrical water phantom is clearly visualized. 


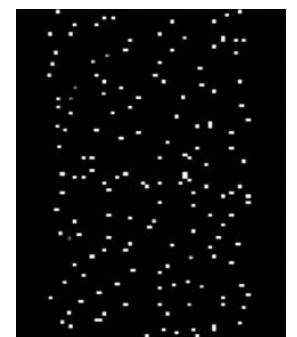

Fig. 8. Projection image for cylindrical shape water phantom.

Note that neither scatter nor attenuation corrections were performed in the reconstruction images. Therefore ringing artifacts can be seen in the reconstruction slices. A conventional filtered back projection algorithm [24] was considered for the preliminary reconstruction images.
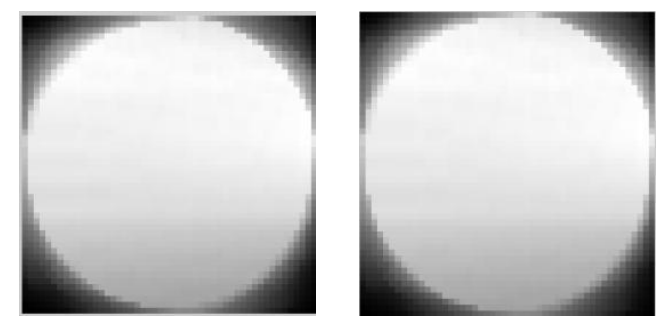

Fig. 8. Reconstructed image of the water phantom.

\section{CONCLUSION}

We simulated a compact design dual head SPECT imaging system with a high performance pinhole array detector module. This system is feasible for high quality SPECT imaging with a large FOV. The compact design of this system is suitable for small laboratory work. Performance evaluation of this system has shown promising results to further investigate and develop it for other experiments. Analysis results based on several pinhole apertures and different source to detector distances have shown the versatility of the system which can be optimized or expanded based on the necessity of different molecular imaging. This compact high performance pinhole array dual head SPECT system allows high precision quantitative analysis along with low scatter radiation and high sensitivity. Therefore, this system could also contribute to fundamental medical research and molecular SPECT imaging.

\section{REFERENCES}

[1] R. J. Jaszczak and R. E. Coleman, "SPECT: Single photon emission computed tomography," IEEE Trans. on Nuclear Science, vol. NS-27, no. 3, June 1980.

[2] D. E. Kuhl, and R. Q. Edwards, "Image separation radioisotope scanning," Radiology, vol. 80, pp. 653-662, 1963.

[3] K. F. Koral, W. L. Rogers, and G. F. Knoll, "Digital tomographic imaging with time-modulated pseudorandom coded aperture and anger camera," J Nucl Med., vol. 16, pp. 402-413, 1975.

[4] A. Jacob, and L. Nourafchan, "Radiological engineering in brain dysfunction imaging processes and neuro informatics," Journal of Nuclear Medicine \& Radiation Therapy, 2013.

[5] A. Rahmim and H. Zaidi, "PET versus SPECT: strengths, limitations and challenges," Nuclear Medicine Communications, vol. 29, pp. 193-207, 2008.

[6] G. A. Kastis, H. B. Barber, H. H. Barrett, S. J. Balzer, D. Lu, D. G. Marks, G. Stevenson, J. M. Woolfenden, M. Appleby, and J. Tueller, "Gamma-ray imaging using a CdZnTe pixel array and a high-resolution, parallel-hole collimator," IEEE Trans. Nucl. Sci., vol. 47, pp. 1923-1927, Dec., 2000.
[7] N. U. Schramm, G. Ebel, U. Engeland, T. Schurrat, M. Béhé, and T. M. Behr, "High-Resolution SPECT Using Multipinhole," IEEE Trans. on Nuclear Science, vol. 50, pp. 315-320, 2003.

[8] F. J. Beekman and B. Vastenhouw, "Design and simulation of a high-resolution stationary," Physics in Medicine and Biology, vol. 49, pp. 4579-4592, 2004.

[9] S. R. Meikle, P. Kench, A. G. Weisenberger, R. Wojcik, M. F. Smith, S. Majewski, S. Eberl, R. R. Fulton, A. B. Rosenfeld, and M. J. Fulham, "A prototype coded aperture detector for small animal SPECT," IEEE Trans. Nuclear Science, vol. 49, pp. 2167-2171, 2002.

[10] Z. Cao, G. Bal, R. Accorsi, and P. D. Acton, "Optimal number of pinholes in multi-pinhole SPECT for mouse brain imaging - A simulation study," Phys. Med. Biol., vol. 50, pp. 4609-4624, 2005.

[11] T. Rahman, M. Tahtali, and M. R. Pickering, "An evaluation to design high-performance pinhole array detector module for four head SPECT: A simulation study," in Proc. SPIE 9212, Developments in X-Ray Tomography IX, 92120K, 2013.

[12] M. T. LeFre, A. A. Vogel, D. L. Kirch, and P. P. Stele, "Seven-pinhole tomography-A technical description," J Nucl Med., vol. 22, pp. 48-54, 1981.

[13] T. Funk, D. L. Kirch, J. E. Koss, E. Botvinick, and B. H. Hasegawa, "A novel approach to multipinhole SPECT for myocardial perfusion imaging," J Nucl Med., vol. 47, pp. 595-602, 2006.

[14] D. W. Austin, M. J. Paulus, S. S. Gleason, R. A. Mintzer, S. B. Siegel, S. D. Figueroa, T. J. Hoffman, and J. S. Wall, "Design and performance of a new SPECT detector for multimodality small animal imaging platforms," IEEE Nuclear Science Symposium Conference Record, vol 5, pp. 3008-3011, 2006.

[15] R.Wojcik, S. Majewski, D. Steinbach and A. G. Weisenberger, "High spatial resolution gamma imaging detector based on 5 ' diameter R3292 hamamatsu PSPMT," IEEE Trans. Nucl. Sci., vol. 45, no. 3, pp. 487-491, June, 1998.

[16] R. Accorsi and S. D. Metzler, "Analytic determination of the resolution-equivalent effective diameter of a pinhole collimator," IEEE Transaction on Medical Imaging, vol. 23, no. 6, June, 2004.

[17] R. Accorsi, F. Gasparini, and R. C. Lanza, "A coded aperture for high-resolution nuclear medicine planar imaging with a conventional anger camera: Experimental results," IEEE Trans. Nucl. Sci., vol. 48, pp. 2411-2417, Dec. 2001.

S. Jan, G. Santin, D. Strul, S. Staelens, K. Assie, and D. Autret, "GATE: A simulation toolkit for PET and SPECT, " Phys. Med. Bio., vol. 49, pp. 4543-4561, 2004

[18] S. Agostinelli, J. K. Allison, A. Amako, J. Apostolakis, H. Araujo, P. Arce, and M. Asai, "GEANT4-A simulation toolkit," Nuclear Instruments and Methods, in Physics Research Section A: Accelerators, Spectrometers, Detectors and Associated Equipment, vol. 506, no. 3, pp. 250-303, 2003

[19] M. V. Green, J. Seidel, J. J. Vaquero, E. Jagoda, I. Lee, and W. C. Eckelman, "High resolution PET, SPECT and projection imaging in small animals," Comput. Med. Imaging Graph., vol. 25, pp. 79-86, 2001.

[20] S. H. Manglos, C. E. Floyd, R. J. Jaszczak, K. L. Greer, C. C. Harris, and R. E. Coleman, "Experimentally measured scatter fractions and energy spectra as a test of monte carlo simulations," Phys. Med. Biol., vol. 32, pp. 335-343, 1987.

[21] K. Kouris, G. A. Clarke, P. H. Jarritt, C. E. Townsend, and S N.Thomas, "Physical performance evaluation of the Toshiba GCA-9300 a triple-headed system," J Nucl Med., vol. 34, pp. $1778-1789,1993$

[22] H. Toyama, A. Higa, M. Yamazato, T. Maehama, R. Ohno, and M. Toguchi, "Quantitative analysis of polarization phenomena in CdTe radiation detectors," Japanese Journal of Applied Physics, vol. 45, no. 11R, pp. 8842, 2006.

[23] P. P. Bruyant, "Analytic and iterative reconstruction algorithms in SPECT," J Nucl Med., vol. 43, no. 10, pp. 1343-1358, 2002.



Tasneem Rahman has received her B.Sc. in electronics and communication engineering from BRAC University, Bangladesh in 2010. She also has completed her MS completion in biomedical engineering and has worked as a research assistant in Impedance Imaging Research Center, Kyung Hee University, Rep. of Korea. Now, she is enrolled as a postgraduate research (Ph.D) student in School of Engineering and Information Technology in UNSW Australia, Canberra, Australia. Her research interests are biomedical imaging, biomedical instrumentation, electrical impedance tomography, SPECT, PET, nuclear medicine and signal processing. She is a member of IEEE and BMPA. 


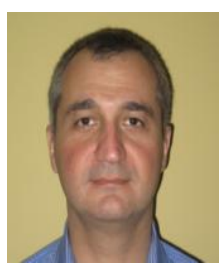

Murat Tahtali graduated from the Middle East Technical University in 1989 with a mechanical engineering degree and in 1993 obtained his masters degree in mechanical engineering on structural vibrations. He migrated to Australia in 1993 and in 1994 joined the University of New South Wales at the Australian Defence Force Academy in Canberra where he is currently a senior lecturer. He obtained his $\mathrm{PhD}$ in electrical engineering in 2008. His main research interests are in adaptive optics, image processing, computed tomography (CT) and GPU computing whilst still maintaining his interest in Mechanical Engineering through teaching.

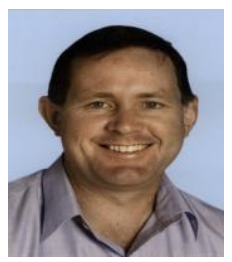

Mark Richard Pickering received the BE degree from the Capricornia Institute of Advanced Education, Rockhampton, Australia, in 1988, and the ME and $\mathrm{PhD}$ degrees from the University of New South Wales, Canberra, Australia, in 1991 and 1995 respectively, all in electrical engineering. He was a lecturer from 1996 to 1999 and a senior lecturer from 2000 to 2009 with the School of Engineering and Information Technology, the University of New South Wales, where he is currently an associate professor. His research interests include video and audio coding, medical imaging, data compression, information security, data networks, and error-resilient data transmission. 